\title{
AirBaltic Case Based Analysis of Potential for Improving Employee Engagement Levels in Latvia through Gamification
}

\author{
Daiga Ērgle \\ University of Latvia
}

\begin{abstract}
In 2014, airBaltic Corporation introduced a gamified electronic platform called Forecaster with a purpose to increase its employee engagement. The article considers the approach used by the organisation, analyses results, advantages and drawbacks of the organisation, and mainly formulates recommendations for the organisation in order to improve impact on employee engagement through gamification. As a result of the research, the author proposes 8 steps that are useful and applicable to any organisation, and as such those may serve a broader purpose than just improving airBaltic Forecaster tool. The objective of the paper is to draw learnings and put forward suggestions for the organisations in Latvia that are concerned with improving their employee engagement and demonstrate how gamification can be helpful in their endeavour.
\end{abstract}

Keywords - Employee engagement, gamification, human resource management.

\section{INTRODUCTION}

In the past few years, gamification has emerged as a trend within the business and marketing sectors, and has recently grabbed the attention of academics, educators and practitioners (Seaborn \& Fels, 2015). At this moment, some of the most common applications of gamification are in the areas of employee performance, innovation management, education, personal development and customer engagement (Seaborn \& Fels 2015). There are different theories written about making gamification attractive and making people feel engaged in the game. Most of these theories are user-centred design theories. This means that the user's needs and goals are the first consideration in designing gamification in order to create meaningful games (Nicholson, 2012). According to Seaborn \& Fels (2015), gamification has been largely, though inconsistently, referred to as the selective incorporation of game elements into an interactive system without a fully-fledged game as the end product (Deterding, Dixon, Khalad, \& Nacke, 2011). This can also be described as the use of game design elements in a non-game context (Deterding, Dixon, Khalad, \& Nacke, 2011). This definition by Deterding, et al. is most widely used by different sources and practitioners. For the purpose of the given research, this definition of gamification is also chosen.

According to a survey of Harvard business review (2013), engagement is by companies seen as a factor most likely to bring success. This places engagement as one of the top business priorities for companies. Of all factors that can drive success, reduce costs and increase revenue and growth, engagement is seen as a key factor with a percentage of $71 \%$ (HBR, 2013).

Experiencing lowering employee engagement several years in a row (measured during annual employee commitment survey since 2011) that followed company restructuring in 2012, in 2014 airBaltic Corporation introduced a gamified electronic platform, called Forecaster, with a purpose to increase its employee engagement by keeping them better informed about business goals as well as by gathering employee feedback in certain forms towards business decisions.

The idea behind the game was to post in the Forecaster platform information about different business related projects, upcoming decisions, business ideas, goals, etc. Posts were called Forecaster Projects, and each project offered employees to voice their opinion, whether they believed in a particular business idea, or upcoming decision was good or not so good, whether a particular goal would be reached or not, etc. They were doing so through buying or selling shares of the specific Project (where buying was a behaviour in case of positive opinion and selling in the case of negative opinion). Certain amount of virtual money, called airBaltic coins, was allocated to each player at the beginning of a game "season" (each game period lasted for about 1-2 months, where winners of the game were announced at the end of such "season" and a new "season" launched). Project "owners", the ones who were posting specific projects, tried to foster discussion and comments around those, to have maximum feedback from the staff and also to provide additional information in cases description turned out to be insufficiently clear. It enabled management to pitch ideas in a simple format, and staff to provide insight in a fun way.

Scope of the study was to consider the approach airBaltic has been using, analyse results of implementing Forecaster, advantages and drawbacks the organisation has experienced, and mainly to draw recommendations for the organisation in order to improve impact on employee engagement through gamification. The aim of this study went beyond the benefit of one particular organisation. It was to draw learnings for other organisations in Latvia that are concerned with improving their employee engagement levels.

\section{Methodology of Research}

Research was mainly conducted within the organisation analysing documents, survey reports and procedures available internally, as well as interviewing employees and managers. Next to quantitative data available from Employee surveys, qualitative data were used to get a deeper understanding about the current situation concerning employee engagement and Forecaster at airBaltic. Interviews were carried out with those employees who were behind Forecaster, with the regular users of the game, as well as with non-users to understand the reasons. 
Theoretical background based on the literature review served the purpose to choose an applicable definition of gamification and employee engagement. It was a necessary step as gamification and employee engagement are constructs used with different definitions. Theoretical research also allowed differentiating engagement with the game from engagement with the company and helped see how one could be used to foster the other. Theoretical research helped create a conceptual framework, which provided a structure for further data collection within the organisation through deep dive interviews. The theory provided a deeper understanding of gamification and how employees could be engaged using Forecaster or other gamified electronic tools. Based on the theory, synthesis of a conceptual framework was described and a plan developed for designing a business game like Forecaster, which would respond to the goal of achieving higher employee engagement in the organisation.

\section{TheORY REVIEW}

As mentioned above, for the purposes of the present research, the author chose a gamification definition by Deterding, Dixon, Khalad \& Nacke (2011) where they described gamification as the use of game design elements in a non-game context.

When designing a business game (just like any other), it is very important to have a deep understanding of users and what motivates them to engage in the game. Bartle (1996) conducted research that contributed to this subject. His research was about player identities in a game called MUD. He pointed out that not all players played for the same reason or played in the same way. This has resulted in the classification of four types of players that are often referred to in the theory about games. The four types can be identified as follows (Bartle, 1996):

Achievers are interested in ACTING on the WORLD. They are typical gamers playing to "win". They give themselves game-related goals and vigorously set out to achieve them. The point of playing is to master the game, and make it do what you want it to do. Achievers are proud of their formal status in the game's built-in level hierarchy, and the little time it took to reach it.

Explorers like INTERACTING with the WORLD. Explorers are interested in having the game surprise them. They delight in discovery. They try to find out as much about the environment's topology and physics. Explorers are proud of their knowledge of the game's finer points, especially if new players treat them as founts of all knowledge.

Socializers are interested in INTERACTING with other PLAYERS. They spend a lot of time chatting, and empathise with other players. Finding out about people and getting to know them are far more worthy than treating them as fodder to be bossed around. The game world is just a setting; it is the characters that make it so compelling. Socializers are proud of their friendships, their contacts and their influence.

Killers like ACTING on other PLAYERS. They wish to dominate them, either through bullying or politicking. They use the tools of the game to cause distress to other players. Killers are proud of their reputation and of their oft-practiced fighting skills.

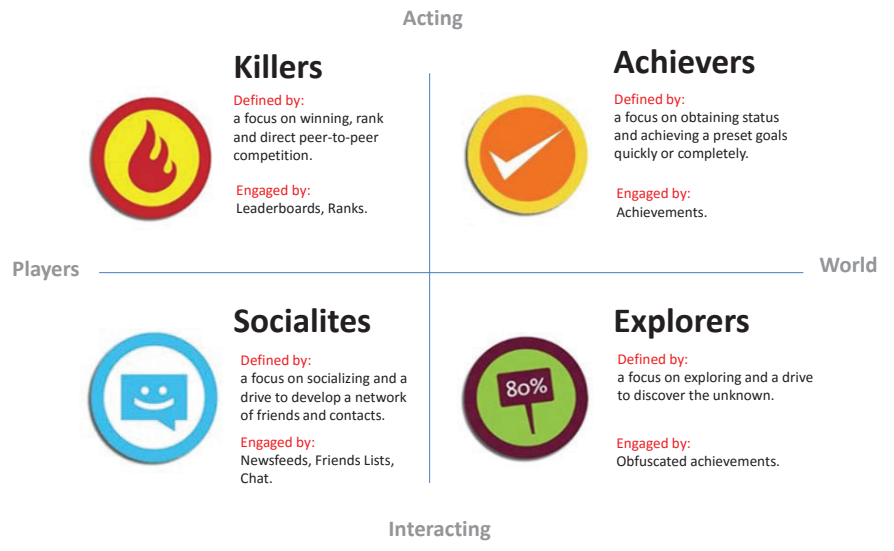

Fig. 1. Bartle's Player Types (Bartle, 1996).

Although in practice Bartle's player types are used in game design, there is some criticism addressed by Dixon (2011). The issue is that the player types were never intended to be a general typology of all digital game players; however, it is often referenced out of MUD context and applied to game design generally, and also recently in gamification. Secondly, the types may be overlapping or mixed, yet Bartle asserts that they are mutually exclusive. Professor of Psychology Mihaly Czikszentmihalyi has designed the theory of flow, which is often referred to in gamification because creating a feeling of flow is important for a game to be successful. According to the theory, flow experiences are those optimal and enjoyable experiences in which we feel "in control of our actions, masters of our own fate...we feel a sense of exhilaration, a deep sense of enjoyment" (Czikszentmihalyi, 1990). Components introduced by him can also be used in gamification to design elements that create flow in a game. Czikszentmihalyi (1990) describes a sense of control over the environment as the most salient element of the flow state. The component of control is very important for gamification. Research on human computer interaction indicated that people find computer games so captivating due to the powerful sense of control these games give their players (Ghani \& Deshpande, 1994).

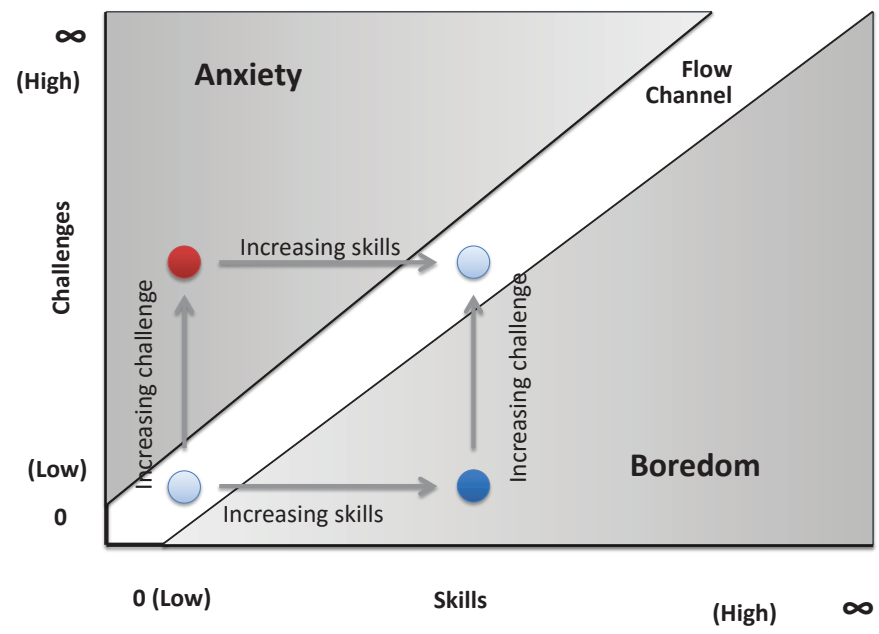

Fig. 2. The state of flow is achieved between anxiety and boredom (Xu, 2011). 
The component of an attainable, balanced goal is also important in gamification. Flow theory suggests that a flow can occur when an activity or a game challenges an individual enough to encourage playful and exploratory behaviours (challenges), without the activity or game being beyond the individual's reach and control (skills) (Czikszentmihalyi, 1990). A task that is not challenging or requires excessive time to complete becomes boring and players lose interest; a task that is too hard causes a lack of control, frustration and anxiety and again players lose interest (Xu, 2011). With a person's skills improving over time, the challenge needs to increase along with the improving skills (Xu, 2011).

When a game is designed, it is important to create a positive and enthusiastic attitude towards it at an organisation to make sure employees actually use gamification. In their research, Hamari and Koivisto (2013) investigated how social motivations predicted attitude towards the use of gamification, and intentions to continue using a gamified service. The results clearly showed that social motivators, in particular social influence of others, clearly predict long-term use and recommendation to others.

These findings underline the importance of a community of people that are committed to the goals the gamification application promotes.

Motivation is very important to engage employees in gamification. Therefore, it is important that a designer knows which motivation to address and which game elements to use. Both extrinsic and intrinsic rewards can be used to motivate employees to engage in certain behaviour; however, in gamification a lot of interest is given to intrinsically motivating employees to play (Seaborn \& Fels, 2015). This might be due to the research of Deci, Koestner, and Ryan (2001) who found that almost all forms of extrinsic rewards reduced intrinsic motivation. The implication of this is that once gamification is used to provide extrinsic motivation, the user's intrinsic motivation decreases (Nicholson, 2012). However, if you first starts using extrinsic rewards and then decides to stop the reward program in order to only concentrate on intrinsic motivation, you will be worse off because users will be less likely to return to the behaviour without the external reward (Deci, Koestner, \& Ryan, 2001).

The most often referred intrinsic motivation theory for gamification designers is a self-determination theory (SDT). The SDT proposes that events and conditions that enhance a person's sense of autonomy, relatedness and competence support intrinsic motivation, whereas factors that diminish this sense undermine intrinsic motivation (Ryan, Rigby, \& Przybylski, 2006). Autonomy is defined as the sense of freedom and will when performing a task. Competence can be seen as the feeling one is faced with challenges they can efficiently and competently participate in. Relatedness is the experience that one is connected to another person. The SDT applied to gamification means that in order to intrinsically motivate employees in gamification the game elements should enhance employee's sense of autonomy, relatedness and competence.

Motivational affordance means that motivation is afforded when the relation between the features of an object (a game) and the abilities and background of a subject (the user) allow the sub- ject (the user) to experience the satisfaction of such needs when interacting with the object (the game) (Deterding, 2011).

In practice, this means that to make a good gamified system like Forecaster the game elements should satisfy the motivational needs of employees. Aparicio et al. (2012) proposed in their research which game elements to use in order to increase intrinsic motivation:

- When increasing autonomy one can use the following game elements: profiles, avatars, macros, configurable interface, alternative activities, privacy control, and notification control.

- When increasing competence one can use the following game elements: positive feedback, optimal challenge, progressive information, intuitive controls, points, levels and leader boards.

- When increasing relatedness one can use: groups, messages, blogs, connection to social networks and chat.

Although the work of Aparicio provides an overview of different game elements divided according to SDT principles, the framework has not been applied and research is still ongoing (Seaborn \& Fels, 2015).

All the different theories used for gamification have one aspect in common. They are all centred on users and how to engage them in using gamification. Although some theories may overlap, each theory has its own focus when it comes to engaging employees in gamification, which makes all the theories valuable for a different reason.

Employee engagement has been defined in many different ways. In the academic literature, a number of definitions have been provided, all with a different focus. The Institute of Employment Studies offers a practical and comprehensive definition of engagement: a positive attitude held by the employee towards the organisation and its values. An engaged employee is aware of business context, and works with colleagues to improve performance within the job for the benefit of the organisation. The organisation must work to develop and nurture engagement, which requires a two-way relationship between an employer and an employee (Institute of Employment Studies, 2004). Based on observations in organisational settings, the author finds this definition rather precisely describing the essence of employee engagement; therefore, it is chosen to serve as the definition of engagement for the purpose of the present research.

According to the research of the Institute of Employment Studies (2004), an employee should show these behaviours in order to be engaged:

- Belief in the organisation;

- Desire to work to make things better;

- Understanding of business context and a "bigger picture";

- Respectful of, and helpful to, colleagues;

- Willingness to "go the extra mile";

- Keeping up to date with developments in the field.

For an organisation it is important to know how to drive the behaviour of an engaged employee. According to the Institute of Employment Studies (2004), the strongest driver of all is a sense of feeling valued and involved. This has several key components that an organisation needs to consider: 
1. Involvement in decision making;

2. The extent to which employees feel able to voice their ideas, and managers listen to these views, and value employees' contributions;

3. The opportunities employees have to develop their jobs;

4. The extent to which the organisation is concerned about employees' health and wellbeing.

Especially the first two key components have the potential to be integrated in workplace gamification. Therefore, employee participation in decision making and voicing ideas through crowd-sourcing were specifically addressed by the Forecaster tool.

Involvement in decision making has some important positive effects that are worth mentioning. Firstly, it can arouse a sense of ownership and commitment of employees to the company (Han, Chiang, \& Chang, 2010). In addition, employees who participate in organisational decision making may think they are more closely connected to organisational goals. Employees themselves can benefit from participation in decision making. It has been argued that through the use of employee participation in decision making employees can learn the art of self-management, cooperation and responsibility. In addition, employees can express talents through participating and satisfy needs of human growth (Han, Chiang, \& Chang, 2010). It therefore has several positive effects on employees within a company.

Online communication channels are very important in order to create employee participation in decision making. These kinds of channels are needed for employees to express their opinion in an easy way.

Furthermore, these online communication channels are also important for managers to obtain suggestions from organisational members when making decisions (Han, Chiang, \& Chang, 2010).

An online opportunity for employees to voice their ideas is through intra-corporate crowd sourcing. Although not a lot of research has been conducted on intra-corporate crowd-sourcing, some researchers have discussed the topic. Intra-corporate crowd-sourcing (ICC) refers to the distributed organisational model used by the firm to extend problem-solving to a large and diverse pool of self-selected contributors beyond the formal internal boundaries of a multi-business firm: across business divisions, bridging geographic locations, levelling hierarchical structures (Villarroel \& Reis, 2010, 1). Through crowd-sourcing an organisation could use the intelligence of the crowd to come up with new ideas for improvement of the company. The main point of crowd intelligence is articulated by Ghafele and Gibert $(2011,8)$ : "The power of crowd-sourcing lies in its ability to draw from a diverse intellectual background where networking technologies link the widest possible range of information, knowledge and expertise." Therefore, an organisation needs these networking technologies as channels and formal selection process for exploiting employee's ideas.

\section{Findings AND Results}

Owing to the theoretical research, the author proposes eight steps for building an effective business game that is part of conceptual framework. The list is derived from different authors who have written about gamification. All authors highlight different steps, which are important when creating gamification in an organisational setting. The first four steps in the list, and also step 7, are derived from Deterding (Gamification: Designing for Motivation, 2012). In this article, he explains some crucial steps that are used by consultants when designing gamification. Aparicio (Analysis and Application of Gamification, 2012) confirms the first 3 steps that Deterding proposes in his research. Step 5 and 6 are also derived from Aparicio et al. (2012). Step 8, which is the last step, is about continually monitoring and improving the game itself. This last step is derived from Robson et al. (2015), who mentioned importance of ongoing monitoring and adjustment of gamification methods.

These 8 steps are as follows:

1. To identify the overall business goal top management is willing to reach;

2. To identify the main objective of gamification: to become aware of the entity you want to gamify in order to reach the business goal;

3. To identify the users: what is in it for them, what motivates them to engage, what is their interest? (to know the stakeholders);

4. To identify the context/culture in which the game will be used;

5. To designing the game and game mechanics: to select game elements that engage a user while accomplishing the business objectives;

6. To create a measurement plan to determine effectiveness and ROI;

7. To implement and communicate the plan;

8. To constantly monitor the effectiveness and added value of the game: to keep adjusting and improving the gamification experience.

The steps above are useful and applicable to any organisation considering an opportunity to increase its employee engagement levels through gamification at work.

The given model was applied to analyse strengths and weaknesses of airBaltic Forecaster tool.

Forecaster was born out of a former start-up project by the current CFO of the company. It was based on a very successful crowd casting website where people could try and predict the outcome of trivial things in the media, like the winner for the Latvian edition of idols. The CFO wanted to duplicate that idea in an organisational setting. The CFO recognised that some employees had specific insights that could predict failure or success of some projects. The first and foremost idea for Forecaster was to harvest these unique insights to prevent harm from low quality decisions.

By selling and buying shares of managers' projects, employees had the opportunity to let managers know if they believed in the project target. An added benefit of Forecaster would be that employees obtained information about what was happening in departments they did not come in contact with. They could learn more about the aviation business and better understand what was going on within the organisation. With these benefits in mind, the CFO pitched Forecaster to the board, and he got permission to run the game. 
Forecaster is about involving employees and communicating to employees about what is going on within airBaltic. This is why the HR department was also enthusiastic about the possibilities of Forecaster from their point of view. They saw opportunities in Forecaster to increase engagement within the company by addressing some top priority weaknesses as seen in the annual employee commitment surveys. HR also saw opportunities to reach generation $\mathrm{Y}$ and $\mathrm{Z}$, "the game generation" much more effectively through the use of Forecaster.

The launch of Forecaster was done via email and intranet communication. It created a buzz within the company and most of the employees who had an account made this account during the launch of Forecaster. In addition, the fresh way of thinking about managerial decision making and internal communication earned airBaltic a lot of external attention and several awards.

However, the current Forecaster can still be seen as a pilot with a lot of potential improvements. CFO and HR are wondering if Forecaster is adding the potential value and what needs to be done to increase this value for management and employees. At present, there is not much sustainable enthusiasm for the game since generating projects from managers to put on Forecaster is an effort every time. From the 327 employees who have an account, there are only 90 active players. This is barely $10 \%$ of the whole company. On average, 15 people log on daily. The group of active players is a homogenous group of employees. These are employees who work behind a computer and have the opportunity to sneak and peak once a week at the game. They are also mostly financial employees and analysts with higher education who work at the head office. However, this group of employees represents only a small part of all employee groups. The largest group (50\%) of employees is flight operation consisting of cabin crew and pilots. This is a group that is more difficult to reach and the members of which do not play Forecaster that much.

The ideal vision of Forecaster according to the CFO is that it will help decision making as employees can share their unique insights and might safeguard management from making ill-advised decisions. Forecaster could then theoretically provide management with new insights and ideas from employees and allow for crowd-sourcing of innovation for new revenue models or ventures.

In addition, it could serve an employer transparency purpose where employees can see what is going on in other departments. The tool could be able to educate employees on airBaltic goals and managerial projects. Next to tangible outcomes such as better decision making, higher engagement or new ventures, Forecaster has the potential to change perception of management and culture of airBaltic. However, at the moment this ideal vision is still far away according to the CFO and HR.

Main question that airBaltic management is looking to answer is: How to design, implement and communicate Forecaster so that it becomes an attractive platform that adds value for airBaltic and has the effect that employees feel more engaged with the company?

Findings are summarised according to the first 4 steps of the above described list of 8 steps.

Interviewing employees and management of airBaltic helped investigate these first steps and come to certain conclusions.
The first 4 steps are about identifying goals, users and context. Information gathered from steps 1-4 allows the company to design, communicate and implement the game (step 5-8). These last steps are an essential part of recommendations for the organisation.

The identified overall business goal for Forecaster tool at airBaltic is to decrease turnover and increase productivity by engaging employees within airBaltic.

Based on interviews, it can be concluded that a majority of employees see more possibilities for Forecaster apart from communication and are also open for these possibilities. Their new ideas for Forecaster also fit with the initiative idea of the CFO about decision making through Forecaster. In addition, the theory has shown that participation in decision making and crowd sourcing, so that employees can give their ideas and opinions, has the potential to increase engagement of employees as employees will feel more involved with the company and its goals. For these reasons, it is believed that these findings can be taken into account as the second entity to gamify next to communication. This means that, based on the findings, two entities to gamify can be considered - communication and idea $\&$ opinion sharing.

Next to identifying the overall business goal and the entity to gamify, it is important to really know your users and potential users before designing a game. To identify the users of airBaltic, a catalogue was made per type of employees interviewed. Managers who post projects on Forecaster, non-players and players were interviewed.

Interviews with the different employee groups and player types make it clear that every group has different needs and different opinions about Forecaster. Some of them play to win. Others just want to observe and see what is new. Other ones want to feel involvement and influence business decisions. And there are people who are seeking for a platform to "stand out" or "shine" by putting forward their ideas. All these differences need to be taken into account when designing a better Forecaster. The challenge is to design Forecaster in such a way that it is attractive for all the different employee groups. Only then Forecaster can generate enough users.

The last element is to identify the context/culture in which the game will be used. A game will never work properly if no attention is paid to the context and culture. When interviewing employees the conversation often went to the Latvian culture as a determining factor for the company culture. Specifically, they described the culture as a limiting factor for explicit sharing of opinions, open feedback and initiative to come up with suggestions. This is therefore an important aspect to consider for Forecaster (as well as for other organisations in Latvia potentially looking at gamified employee engagement solutions). On the another hand, these characteristics of culture, if true, could be another reason why a game like Forecaster may succeed better than any other platforms intended for gathering employee feedback, since it offers opportunity for relatively anonymous participation in case someone prefers to hide behind the username, as well as the game like this offers a fun element, which may make it easier to come up with ideas. If those are not accepted - then it is just a game and does not make an employee to feel like failed. 
According to the expert interviews, one should never see gamification as just IT implementation. It is not only about creating an online environment but also about creating an offline environment.

In this offline environment employees need a certain reason why to go to Forecaster. If the company wants employees to share ideas, provide feedback through Forecaster (or any gamified engagement platform), it has to develop the idea sharing culture in the offline world.

Based on the research, it can be concluded that Forecaster in its current form will not have a lasting effect and therefore cannot have the intended long-term effect on engagement. Key elements have to change in order to improve Forecaster. The first recommended option would be a fully redesigned Forecaster with all elements in line with the 8-step model that could foster employee engagement according to findings. Forecaster's new version would not only be able to increase engagement but could also create possibilities for more innovation, collaboration and knowledge sharing.

Findings suggested that employees would like to see Forecaster as a platform where idea and opinion sharing would be made possible. Therefore, Forecaster needs to be designed with these elements as the focus. New edition of Forecaster should be seen as a game in which it is possible for employees to be actively involved in significant organisational issues through sharing ideas, knowledge, information and opinion. When employees feel they can share their opinion, questions and ideas, they will feel more engaged and involved with the company. It is also a strong signal to employees that the management values their input and wants them to be able to voice their opinion. In addition, the top management can benefit from the good ideas and useful insights of employees in order to make better decisions and implementing innovative ideas.

\section{CONCLUSION}

Although Forecaster can have a significant impact and increase engagement, this does not mean that it is going to solve all engagement weaknesses. Therefore, it is recommended both for airBaltic Corporation and other companies in Latvia to investigate closer other causes of low employee engagement, and, accordingly, to consider other solutions that address those issues. Gamification of people management processes can potentially be highly valuable in increasing employee engagement. However, airBaltic Forecaster in its current form needs to be changed in order to have more impact on employee engagement. Learnings from airBaltic experience are helpful for other organisations that look for solutions to increase their employee engagement. At the moment, Forecaster is not living up to its full potential. Therefore, the question arose during research if it were wise to discontinue it. After interviewing a large number of employees, it is, however, highly recommended not to do so. Forecaster has not have a fair fighting chance to prove itself yet, since it has only been running for 10 months with minimal resources and support. Notwithstanding this minimalistic approach, employees already value it because of the information they would otherwise never get. To that respect, Forecaster is a successful information tool. It has a number of fans and is successful at a smaller scale that could quite easily be up scaled. Discontinuing would set a bad example because sharing information and knowledge is of crucial importance to engage employees. The signal airBaltic gives by discontinuing could be demotivating. A gamified system needs attention, support and time, and if airBaltic is willing to provide this, Forecaster has all the potential to become successful. Therefore, the recommendations have shown that there are two scenarios how to improve the business game. One scenario would be more oriented towards quick fixes and the other would be a new redesigned game in which the focus would be idea, knowledge and opinion sharing. Due to the number of resources that are needed to create a new game, it was first recommended to the company to do the quick fixes in order to improve the current form of Forecaster and to give it a fair chance. Forecaster needs new life blown into it to create engagement, more content and players. The quick fixes can make this in a cheap, easy way in the short term. Efforts taken for the current game can only be beneficial to the next generation of the application. Popularity of Forecaster in its current form can only create momentum and enthusiasm for sharing and playing with co-workers. After another year the effect can be reviewed and based on these effects a decision on how to proceed can be taken. By doing quick fixes first and a new version second, the game would gradually and naturally evolve and improve, which gives the players the chance to evolve with it and keep attractiveness of the game. Designing of improved game could be done in-house to make it precisely customised to airBaltic. But it could also be outsourced to a third-party. There are solution providers who would mostly have on the shelf solutions but the advantage would be that airBaltic can focus only on rolling the game out to the user and not on system maintenance and design.

In case the organisation is developing a similar solution from the scratch, it would be highly advised to outsource the whole process to a professional firm, unless the company has freely available in-house competence for designing and developing such type of a game.

Through the effective use of Forecaster, the flow of communication about strategy, plans and direction of the company can be made better. In addition, the open transparency of top management on Forecaster can improve the perception that employees have of the management. If employees see that management is willing to involve employees in organisational issues and value their involvement this will improve their image and reliability as a good management team. This shows that putting time and effort in Forecaster can directly and positively affect the priority drivers that hinder employee engagement at airBaltic - knowledge about the strategy and goals, and perception of management working as an efficient team.

In addition, gamification of people management processes has the ability to attract and engage the young generation. Generation $\mathrm{Y}$ and $\mathrm{Z}$ are generations that want to be challenged, they want to contribute and they want to be able to participate and impact organisational issues. Especially, the new and improved design of Forecaster would meet these expectations because of new features that would help to gain confidence that their ideas and expertise are valued. If any organisation is willing to 
specifically address the young generation and future employees, then properly designed gamification of people management processes may be of a great value.

Forecaster and similar solutions seem to be games for the future because the world is changing too fast for one person to know everything. The knowledge of the crowd is paramount to keep up with change. Top management cannot and will not know everything to make the company successful, while some of the 1200 employees in airBaltic case might have the most brilliant ideas. If the organisation invests the effort to recruit the best candidates and train them to be even better, it should also make an effort to listen to them.

If, in addition to better business decisions ensured through employee participation, the organisation also reaches higher employee engagement levels that positively influence profitability, innovativeness and competitiveness, this is an effort worth investing into. The analysis of airBaltic experience with Forecaster tool, as well as the theoretical research suggests that this is the case. As discussed in the theory review, a game environment has elements that are better than any other frameworks known in business secure player's engagement with the game. Business organisations, learning from game settings, may come up with solutions that through these elements foster employee engagement with the company.

\section{REFERENCES}

airBaltic Intranet. (2015). about airBaltic. Retrieved from Intra.Airbaltic.com. Aparicio, A., Vela, F., Sánchez, J., \& Montes, J. (2012). Analysis and application of gamification. Elche, Alicante, Spain. http://dx.doi. org $/ 10.1145 / 2379636.2379653$

Badgeville. (2015). Game Mechanics. Retrieved from Badgeville.com: https:// badgeville.com/products/gamification

Bartle, R. (1996, August 28). HEARTS, CLUBS, DIAMONDS, SPADES PLAYERS WHO SUIT MUDS. Colchester, Essex, United Kingdom.

Bunchball. (2015, June 1). Nitro Gamification Platform. Retrieved from Bunchball.com: http://www.bunchball.com/products/nitro

Cook, W. (2013). 5 reasons you can't ignore gamification. Newton: Inward Strategic Consulting.

Czikszentmihalyi, M. (1990). The psychology of optimal experience. New York: Harpen \& Row.

Deci, E., Koestner, R., \& Ryan, R. (2001). Extrinsic rewards and intrinsic motivations in education: reconsidered once again. Review of Educational Research, 1-27. http://dx.doi.org/10.3102/00346543071001001

Deterding, S. (2011). Situated motivational affordances of game elements: a conceptual model. Vancouver, Canada: CHI 2011

Deterding, S. (2012). Gamification: designing for motivation. Interactions, 14-17. http://dx.doi.org/10.1145/2212877.2212883

Deterding, S., Dixon, D., Khalad, R., \& Nacke, L. (2011, September 2830). From game design elements to gamefulness: defining Gamification. Mindtrek. http://dx.doi.org/10.1145/2181037.2181040

Dixon, D. (2011). Player types and gamification. Vancouver: CHI.

Fogg, B. (2009). A behavior model for persuasive design. California: Persuasive Technology Lab, Stanford University. http://dx.doi. org/10.1145/1541948.1541999

Ghafele, R., \& Gibert, B. (2011). Crowdsourcing patent application review: leveraging new opportunities to capitalize on innovation? Intellectual Property Quarterly, 23-33, No. 3.

Ghani, J., \& Deshpande, S. (1994). Tasks characteristics and the experience of optimal flow in human-computer interaction. The journal of psychology, 381-391. http://dx.doi.org/10.1080/00223980.1994.9712742

Hamari, J., \& Koivisto, J. (2013). Social motivations to use gamification: an empirical study of gamifying exercise. Proceedings of the 21st European Conference on information systems. Utrecht.

Han, T., Chiang, H., \& Chang, C. (2010). Employee participation in decision making, psychological ownership and knowledge sharing: mediating role of organizational commitment in Taiwanese high-tech organizations. The International Journal of Human Resource Management, 21(12), 2218-2233.

Harvard business school analytical review. (2013). The impact of employee engagment on performance. London: Harvard business review publisher.

Higgins, A. (2014, July 16). 3 Examples of gamification in the workplace. Retrieved from Concur.com: https://www.concur.com/blog/en-us/3-examples-of-gamification-in-the-workplace
Huatari, K., \& Hamari, J. (2012). Defining gamification - a service marketing perspective. Presented at MindTrek'12. (pp. 17-22). Proceedings of the 16 the International Academic Mind Trek Conference.

Institute of Employment Studies. (2004). Report Summary: The drivers of employee engagement. Retrieved July 7.

Nicholson, S. (2012). A User-centered theoretical framework for meaningful gamification. Games + learning + Society (pp. 1-7). Madison, WI: School of Information Studies, Syracuse University

Robson, K., Plangger, K., Kietzmann, J., McCarthy, I., \& Pitt, L. (2015). Is it al a game? Understanding the principles of gamification. Elsevier.

Ryan, R., Rigby, C., \& Przybylski, A. (2006). The Motivational Pull of Video Games: A Self Determination Approach. Motiv Emot, Springer Science, 347-363.

Seaborn, K., \& Fels, D. (2015). Gamification in theory and action: Asurvey. International Journal of Human-Computer Studies, 74, 14-31. http://dx.doi. org/10.1016/j.ijhcs.2014.09.006

Shayon, S. (2013b, June 13). Gamification with new aviation empire game. Retrieved from Brandchannel.com: http://brandchannel.com/2013/06/13/ $\mathrm{klm}$-readies-full-on-social-gamification-with-new-aviation-empire-game/

Speckund. (2015). Gartner Hype Cycle of Emerging Technologies, Hypes und Trends für 2014 / 2015. Retrieved May 8, 2015, from speckund.ch: http:// speckund.ch/2/gartner-hype-cycle-emerging-technologies-2014-2015/

TNS. (2015). airbaltic corporation TRI*M Employee Commitment Survey. Riga: TNS.

Villaroel, J., \& Reis, F. (2011). A stock Market Approach to Online Distributed Innovation: the Trade-off between Speculation and Innovation Performance. Workshop on social computing and user generated content ACM-EC'11, (pp. 1-5). San Jose.

Werback, K. (2014). (Re)Defining Gamification: A Process Approach. Persuasive Technology: Lecture Notes in Computer Science, pp. 266-272. http:// dx.doi.org/10.1007/978-3-319-07127-5 23

$\mathrm{Xu}, \mathrm{Y}$. (2011). Literature review on web application gamification and analytics. Honolulu: Collaborative Software Development Lab, University of Hawai.

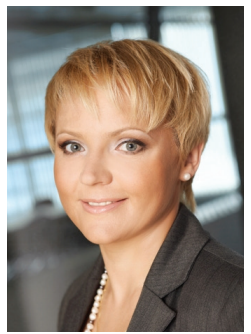

Daiga Ērgle is a PhD student at the University of Latvia, Faculty of Economics and Management. She holds an MBA degree from RTU Riga Business School, obtained in 2003. In 1996, she received a professional degree in Foreign Economic Affairs Management from the University of Latvia and in 1989 a qualification of large Cultural Events Manager from the Latvian State Conservatory.

Since 2010, D. Ërgle has been a Senior Vice President of Human Resources at airBaltic Corporation in Riga, Latvia. Prior to this, she was leading an Executive Search branch of the Human Resource Consulting Company Fontes. Earlier, Daiga Ërgle worked as a Human Resource Manager at such organisations as Stockmann, Siemens and Gutta. In parallel to the above-mentioned activities, since 2008 D. Ërgle has been teaching Human Resource Management for MBA students at RTU Riga Business School. The major field of her research interest is human resource management in general and employee engagement in particular. She is a member of the Latvian Association for Personnel Management and a founding member of HR Club in Latvia.

E-mail: daiga.ergle@airbaltic.lv 